\title{
Computational Fluid Dynamics Simulation of High Speed Jet Under Different Input Pressures
}

\author{
Jie Gong, Wen Xia ${ }^{*}$,Ji-Hua Li, Xiao-Yi Wei \\ Chinese Agricultural Ministry Key Laboratory of Tropical Crop Production Processing, Agricultural Product Processing Research Institute at \\ Chinese Academy of Tropical Agricultural Sciences, Zhanjiang, China \\ Email address: \\ rosegongjie@hotmail.com (Jie Gong), xiawensg@163.com (Wen Xia), foodpaper@126.com (Ji-Hua Li),weixywei@163.com (Xiao-Yi Wei) \\ ${ }^{*}$ Corresponding author
}

\section{To cite this article:}

Jie Gong, Wen Xia, Ji-Hua Li, Xiao-Yi Wei. Computational Fluid Dynamics Simulation of High Speed Jet Under Different Input Pressures. International Journal of High Energy Physics. Vol. 4, No. 1, 2017, pp. 12-18. doi: 10.11648/j.ijhep.20170401.12

Received: March 13, 2017; Accepted: April 6, 2017; Published: May 2, 2017

\begin{abstract}
The aim of this study is to execute the computational fluid dynamics (CFD) simulation of high speed jet under different input pressures (i.e., 80, 120, 160, 200, and $240 \mathrm{MPa}$ ). In particular, this study focuses on the pressure distributions and streamlines of the orifice in high speed jet, primarily because the orifice plays a role in accelerating the flow of liquid, having significant effects on the working performance of high speed jet. Firstly, the two-dimensional geometric model of high speed jet is established on the basis of the actual operational conditions. Next, the unstructured grids of high speed jet are generated by means of ICEM CFD 16.0. Virtually, the computational fluid dynamics simulation of high speed jet is a two-phase flow (gas-liquid) problem, so the homogeneous (Eulerian-Eulerian) two-phase model is employed to carry out the gas-liquid interaction. Particularly, the turbulent flow computation of high speed jet is carried out with procedures based on the Reynolds-averaged Navier-Stokes (RANS) equations. As the flow of high speed jet is highly turbulent, the RNG k- $\varepsilon$ turbulence model derived by Yakhot et al. (1992) is utilized in this study. Finally, the computational fluid dynamics (CFD) simulation of high speed jet is implemented by using the CFX-Solver in ANSYS CFX 16.0. The simulation results show that when liquid flows through the orifice, the pressure of flows decreases swiftly, whereas the velocity of flows skyrockets to the maximum value and then decreases slightly. In addition, the relationship between the working pressure and input pressure and the relationship between the working velocity and input pressure are achieved, which could provide certain theoretical guidance for predicting the working pressure and velocity of high speed jet based on real input pressures.
\end{abstract}

Keywords: Computational Fluid Dynamics, Finite Volume Method, Gas-Liquid Two Phase, High Speed Jet (HSJ), Input Pressure, Unstructured Grids, Reynolds-Averaged Navier-Stokes (RANS) Equations, RNG Turbulence Model

\section{Introduction}

High speed jet (HSJ) is a novel device combination with high pressure and speed [1-3]. Actually, high speed jet takes advantage of the water as the medium. With the help of the hydraulic equipment, the aqueous medium can be pressurized to a certain pressure, and then the aqueous medium is jetted via the nozzle, generating a steam of high speed, high energy, and high penetrating power jet stream known as 'water jet scalpel'. In fact, water jet technology is a category of independent and complete technology, whereas this technology developed rapidly during past 30 years. As a result of its unique cool grinding and excellent performance, water jet technology could develop into the cutting technique, crushing technology, and cleaning technology through changing a range of parameters (e.g., pressure, flow, power, etc.). Particularly, the schematic diagram of high speed jet is shown in Figure 1 [1-3]. As shown in Figure 1, high speed jet involves a piston chamber where crude suspension is drawn into through the downward stroke [1-3]. Virtually, the suspension is forced to pass through a minor fixed nozzle, whose role is to produce a relatively high speed jet during the upward stroke. Before colliding on the target, the jet moves via a pipe with the varying cross sectional area, and then high speed jet is cooled by a re-circulating flow of coolant from the 
walls of the disruption chamber. More importantly, the available orifice diameters are in the range between 0.1 and $0.18 \mathrm{~mm}$, and the input pressure ranges from 0 to $270 \mathrm{MPa}$, higher than different treatments of high pressure (HP) technology, like dynamic high pressure microfluidization (DHPM) [1-3]. Currently, high speed jet is widely used in various fields, such as the cell disruption, emulsification, refinement of latex particles, etc.

In recent years, although there are a large number of published reports that have focused on the use of high speed jet, the investigations regarding the computational fluid dynamics simulation of high speed jet are rarely studied [1-5]. Computational fluid dynamics, commonly known by the acronym 'CFD', is the analysis of systems involving fluid flow, heat transfer and associated phenomena such as chemical reactions by means of computer-based simulation $[6,7]$. Through the computational fluid dynamics, the pressure and velocity distribution of the fluid in high speed jet can be observed visually. This study is an attempt to analyze the fluid field of high speed jet under different input pressures by means of the computational fluid dynamics simulation, aiming at providing a range of valuable theoretical guidance for studying the operating mechanism of high speed jet. Also, this study could provide certain reference for the optimization design of high speed jet.

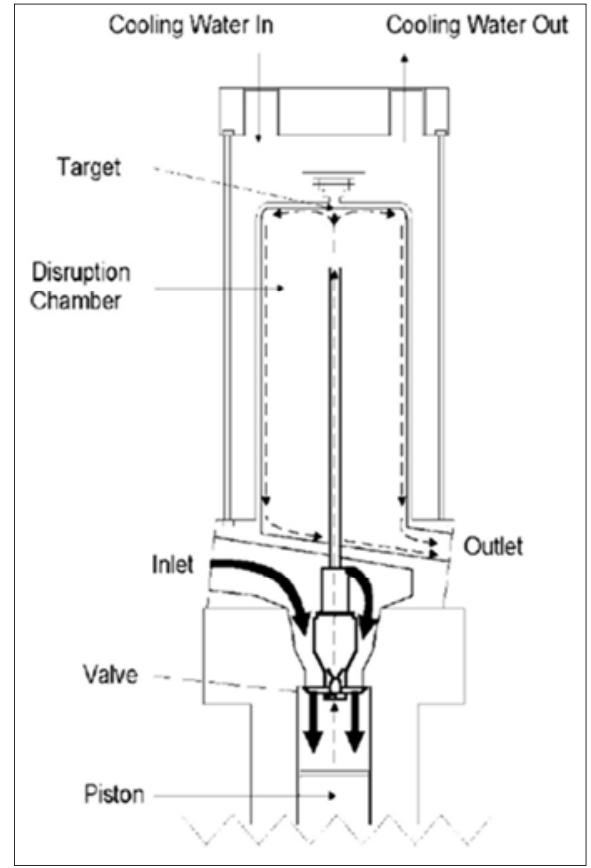

Figure 1. The schematic diagram of high speed jet.

\section{Computational Fluid Dynamics Modelling}

\subsection{Geometry Model}

Undoubtedly, the geometric model is the foundation of the numerical simulation [8]. In general, the real structure of high speed jet includes a number of fine features, like small round and chamfer. If these fine features are considered in the mesh generation, to simulate these features exactly, numerous fine meshes are required, delaying the resolving time. For the convenience of the mesh generation and numerical simulation, a variety of detailed information of the model component should be not taken into consideration. The three-dimensional computational fluid dynamics simulation is extremely time-consuming. Compared with the three-dimensional computational fluid dynamics simulation, the two-dimensional computational fluid dynamics simulation can save plenty of computational cost (e.g., the simulation time, memory capacity, hard drive capacity, etc.). In terms of the structure feature of high speed jet, the two-dimensional computational fluid dynamics simulation can also reflect the fluid field of high speed jet evidently. Consequently, the two-dimensional computational fluid dynamics model of high speed jet is established in this study. Based on the actual operational conditions of high speed jet, the two-dimensional geometric model is simplified. The simplified geometric model of high speed jet is built by Autodesk Inventor Professional according to the entity of high speed jet, as shown in Figure 2. In particular, the orifice diameter in Figure 2 is $0.18 \mathrm{~mm}$.

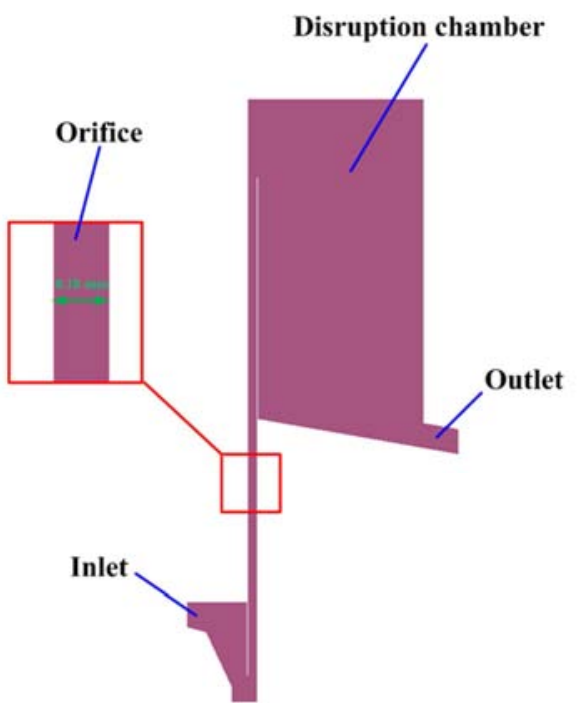

Figure 2. Two-dimensional geometric model of high speed jet.

\subsection{Mesh Generation}

The computational fluid dynamics simulation of high speed jet is carried out via ANSYS CFX 16.0. ANSYS CFX is a high-performance computational fluid dynamics (CFD) software tool that delivers reliable and accurate solutions quickly and robustly across a wide range of CFD and multi-physics applications. At present, ANSYS CFX is recognized for its outstanding accuracy, robustness and speed with rotating machinery worldwide. In reality, the discretization approach used in ANSYS CFX is the finite volume method. The finite volume method takes advantage of the integral form of the conservation equations as its start point $[6,7]$. The solution domain is subdivided into a finite 
number of contiguous control volumes, and the conservation equations are applied to each control volume [6, 7]. In particular, the finite volume method has the ability to accommodate any category of grid. As a consequence, the finite volume method is suitable for complicated geometries $[6,7]$.

ICEM CFD 16.0, a professional mesh generation software, is employed to generate the mesh of high speed jet. Compared with structured grids, unstructured grids allow the calculation of flows in or around geometrical features of arbitrary complexity without having to spend a long time on mesh generation and mapping [6]. Accordingly, unstructured grids are generated in this study. To ensure the accuracy of the numerical simulation, let the minimum mesh size be $0.1 \mathrm{~mm}$. In addition, unstructured grids of high speed jet are shown in Figure 3.

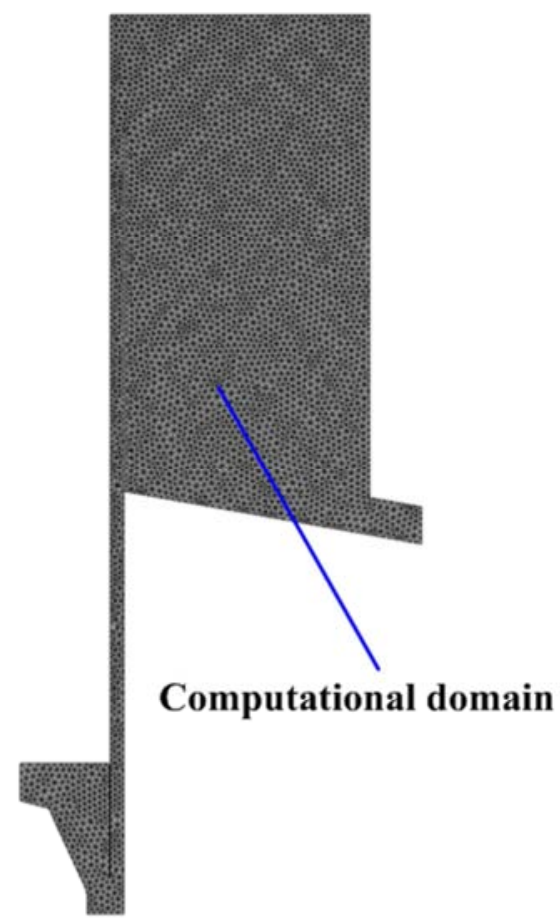

Figure 3. Mesh generation of high speed jet.

\subsection{Theoretical Model}

In actual work, the aqueous medium emerging from the inlet discharges into the empty space of the disruption chamber, as shown in Figure 1 [1]. As a consequence, the jet is taken into consideration as a two-phase (gas-liquid), the system with the liquid jet expanding through the air space of the chamber. There are a number of methods to computing two-phase flows, and the continuous phase fluid is always treated by the Eulerian method [7, 9]. For the universality of the research, water and air are taken as research objects. In this study, assume that the water and air are incompressible and isothermal. In particular, the homogeneous (Eulerian-Eulerian) two-phase model is employed to implement the gas-liquid interaction.

In nature, the fluid flowing state has two main sorts, namely, the laminar flow and turbulent flow. In particular, most flows encountered in engineering practice are turbulent flow [7]. Presently, the vast majority of turbulent flow computations have been carried out with procedures based on the Reynolds-averaged Navier-Stokes (RANS) equations [6]. In consequence, the considered calculation in this study is executed by means of Reynolds-averaged Navier-Stokes (RANS) equations. The following equations are the turbulent flow equations for incompressible flows.

Continuity for the mean flow [10-12]:

$$
\overline{\operatorname{div} \mathrm{u}}=\operatorname{div} \mathrm{U}=0 \frac{n !}{r !(n-r) !}
$$

The time-average $\mathrm{x}$-momentum equation is [10-12]

$$
\begin{aligned}
\frac{\partial U}{\partial t}+\operatorname{div}(U \mathrm{U})=- & \frac{1}{\rho} \frac{\partial P}{\partial x}+v \operatorname{div}(\operatorname{grad}(U)) \\
& +\frac{1}{\rho}\left[\frac{\partial\left(-\rho \overline{u^{2}}\right)}{\partial x}+\frac{\partial\left(-\rho \overline{u^{\prime} v^{\prime}}\right)}{\partial y}+\frac{\partial\left(-\rho \overline{u^{\prime} w^{\prime}}\right)}{\partial z}\right]
\end{aligned}
$$

The time-average y-momentum equation is given by [10-12]

$$
\begin{aligned}
\frac{\partial V}{\partial t}+\operatorname{div}(V \mathrm{U})=- & \frac{1}{\rho} \frac{\partial P}{\partial y}+v \operatorname{div}(\operatorname{grad}(V)) \\
& +\frac{1}{\rho}\left[\frac{\partial\left(-\rho \overline{u^{\prime} v^{\prime}}\right)}{\partial x}+\frac{\partial\left(-\rho \overline{v^{2}}\right)}{\partial y}+\frac{\partial\left(-\rho \overline{v^{\prime} w^{\prime}}\right)}{\partial z}\right]
\end{aligned}
$$

The time-average z-momentum equation yields [10-12]

$$
\begin{aligned}
\frac{\partial W}{\partial t}+\operatorname{div}(W \mathrm{U})= & -\frac{1}{\rho} \frac{\partial P}{\partial z}+v \operatorname{div}(\operatorname{grad}(W)) \\
& +\frac{1}{\rho}\left[\frac{\partial\left(-\rho \overline{u^{\prime} w^{\prime}}\right)}{\partial x}+\frac{\partial\left(-\rho \overline{v^{\prime} w^{\prime}}\right)}{\partial y}+\frac{\partial\left(-\rho \overline{w^{\prime 2}}\right)}{\partial z}\right]
\end{aligned}
$$

The extra turbulent stresses are defined to clarify their structure. In practice, they result from six additional stresses: three normal stresses

$$
\tau_{x x}=-\rho \overline{u^{\prime 2}} \quad \tau_{y y}=-\rho \overline{v^{\prime 2}} \quad \tau_{z z}=-\rho \overline{w^{\prime 2}}
$$

and three shear stresses

$$
\tau_{x y}=\tau_{y x}=\overline{-\rho u^{\prime} v^{\prime}} \quad \tau_{x z}=\tau_{z x}=-\overline{\rho u^{\prime} w^{\prime}} \quad \tau_{y z}=\tau_{z y}=-\overline{\rho v^{\prime} w^{\prime}}
$$

Particularly, these extra turbulent stresses are called the Reynolds stresses, and the equation set (1) and (2-4) is called the Reynolds-average Navier-Strokes equations.

The time-average transport equation for scalar $\varphi$ is given by [10-12]

$$
\begin{aligned}
\frac{\partial \Phi}{\partial t}+\operatorname{div}(\Phi U)=\frac{1}{\rho} \operatorname{div}( & \left.\Gamma_{\Phi} \operatorname{grad} \Phi\right) \\
+ & {\left[-\frac{\partial \overline{u^{\prime} \varphi^{\prime}}}{\partial x}-\frac{\partial \overline{v^{\prime} \varphi^{\prime}}}{\partial y}-\frac{\partial \overline{w^{\prime} \varphi^{\prime}}}{\partial z}\right]+S_{\Phi} }
\end{aligned}
$$


but

$$
\begin{aligned}
& C_{1 \varepsilon}=1.42 \\
& C_{2 \varepsilon}=1.68
\end{aligned}
$$

and

$$
\begin{gathered}
C_{1 e}^{\cdot}=C_{1 e}-\frac{\eta\left(1-\eta / \eta_{0}\right)}{1+\beta \eta^{3}} \\
\eta=\frac{k}{\varepsilon} \sqrt{2 S_{i j} \cdot S_{i j}} \\
\eta_{0}=4.377 \\
\beta=0.012
\end{gathered}
$$

where $k$ is the turbulent kinetic energy, $\varepsilon$ is the dissipation rate of turbulent kinetic energy, $\tau_{i j}$ is the Reynolds stress tensor, $S$ is the mean rate of strain, $\alpha_{k}$ is the turbulent Prandtl number of the turbulent kinetic energy $k, \alpha_{e}$ is the turbulent Prandtl number of the dissipation rate $\varepsilon, \delta_{i j}$ is the Kronecker delta, $C$ is a dimensionless constant, $C$ and $c_{v}$, are the constants determined from benchmark experiments, $\mu$ is the molecular viscosity, $\mu_{t}$ is the dynamic turbulent viscosity, $\eta$ is the dimensionless shear rate, $\eta_{0}$ is the fixed point, and $\beta$ is the constant.

\subsection{Boundary Conditions and Calculations for Solution}

In fact, all CFD problems are defined in terms of initial and boundary conditions [17-19]. Generally, 5 levels of the input pressures of $80,120,160,200,240 \mathrm{MPa}$ are frequently utilized in an attempt to carry out the experimental analysis [2-5]. Thus, the input pressures of 80, 120, 160, 200, and 240 $\mathrm{MPa}$ are set as inlet boundary conditions at the position of the inlet shown in Figure 2, respectively, and the category of inlet boundary conditions is the total pressure. Additionally, outlet boundary conditions are set at the position of the outlet shown in Figure 2, and the group of the outlet boundary conditions is the static pressure with $0 \mathrm{MPa}$.

with

$$
\tau_{i j}=-\rho \overline{u_{i} u_{j}^{\prime}}=2 \mu_{t} S_{i j}-\frac{2}{3} \rho k \delta_{i j}
$$

and

$$
\begin{gathered}
\mu_{e f f}=\mu+\mu_{t} \\
\mu_{t}=\rho C_{\mu} \frac{k^{2}}{\varepsilon}
\end{gathered}
$$

and

$$
\begin{array}{r}
C_{\mu}=0.0845 \\
\alpha_{k}=\alpha_{\varepsilon}=1.39
\end{array}
$$

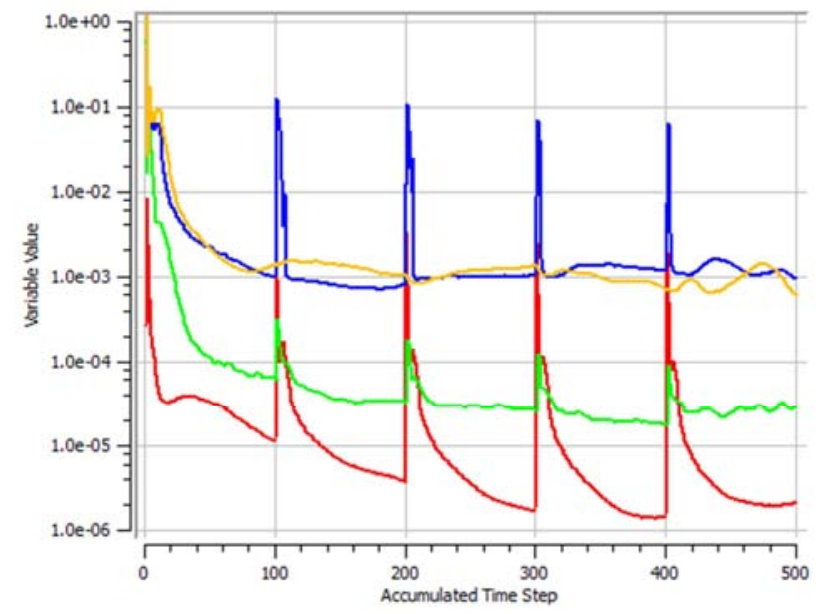

Figure 4. Residual RMS Error values for five successive numerical solutions. 
The analysis type of this study is the steady state. CFX-Solver is used to execute the computational fluid dynamics simulation of high speed jet under different input pressures. In all numerical simulations, the residual RMS Error values have reduced to an acceptable value of 10-6. Presented in Figure 4 are the residual RMS Error values for five successive numerical solutions.

\section{Results and Discussion}

The function of the orifice in high speed jet is to accelerate the flow of liquid. There is no doubt that the flow situation of liquid in the orifice has significant influences on the working performance of high speed jet. Thus, this study pays more attention to the computational fluid dynamics simulation of the orifice in high speed jet.

\subsection{Influences of Input Pressures on Pressure Distribution}

Figure 5 shows the pressure distributions of high speed jet under different input pressures (i.e., 80, 120, 160, 200, and $240 \mathrm{MPa}$ ). According to Figure 5, it is notable that the pressure of flows in the inlet remains at the same level, whereas when liquid flows into the orifice, the pressure of flows decreases swiftly. In addition, the pressure of flows in the disruption chamber basically keeps unchanged. From Figure 5, it is known that the maximum pressure of flows in the orifice is at the entrance of the orifice, and the minimum pressure of flows in the orifice is at the exit of the orifice.

Actually, the pressure of flows at the exit of the orifice is the working pressure of high speed jet. The working pressures of high speed jet under different input pressures are indicated in Figure 6. As indicated in Figure 6, with the rise in input pressures, the working pressure of high speed jet increases from 11.75 to $39.39 \mathrm{MPa}$ between the input pressures of 80 and $240 \mathrm{MPa}$. The curve fitting toolbox in MATLAB is used to fit the curve shown in Figure 6. According to the variation tendency of the curve in Figure 6, the linear approach is selected. Through the curve-fitting technique, the relationship between the working pressure of high speed jet and input pressure is obtained, as illustrated in Eq. (22).

$$
y_{w p}=0.1754 x_{i p}-1.136
$$

where $y_{w p}$ is the working pressure of high speed jet in $\mathrm{MPa}$, and $x_{i p}$ is the input pressure in $\mathrm{MPa}$.

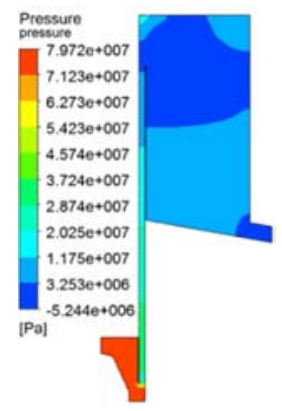

a) $80 \mathrm{MPa}$

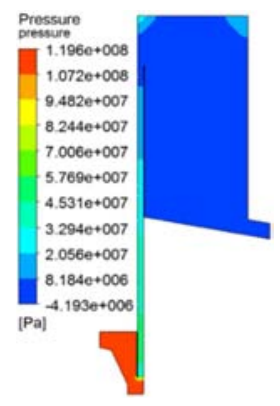

(b) $120 \mathrm{MPa}$

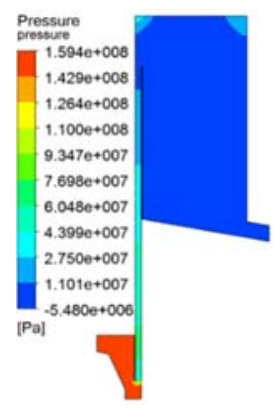

(c) $160 \mathrm{MPa}$

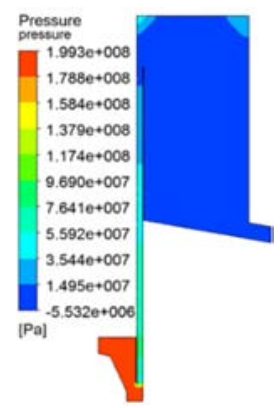

(d) $200 \mathrm{Mpa}$

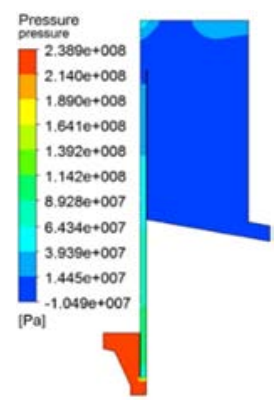

(e) $240 \mathrm{MPa}$

Figure 5. Pressure distributions of high speed jet under different input pressures.

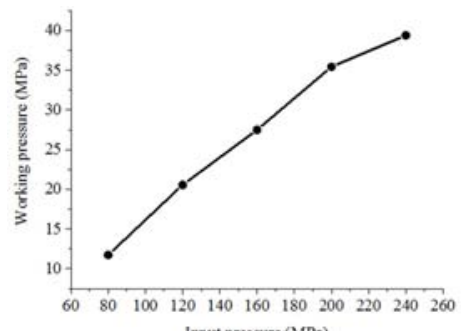

Figure 6. Plot of working pressure versus input pressure. 


\subsection{Influences of Input Pressures on Velocity Distribution}

Figure 7 shows the streamlines of high speed jet under different input pressures. In Figure 7, the velocity of flows goes up slightly within the inlet. However, when flows reach the entrance of the orifice, the velocity of flows soars to the maximum value. More interestingly, the velocity of flows drops slowly from the entrance to exit of the orifice, showing the excellent acceleration performance of the orifice. From the exit of the orifice to the outlet, the velocity of flows plummets.

Similarly, the velocity of flows at the exit of the orifice is the working velocity of high speed jet. The working velocity of high speed jet under different input pressures are illustrated in Figure 8.

As shown in Figure 8, there is an increase in the working velocity from 114.1 to $194.8 \mathrm{~m} / \mathrm{s}$ between the input pressures of 80 and $240 \mathrm{MPa}$. According to the chang+e trend of the curve in Figure 8, the linear method is employed. By using the curve-fitting approach, the relationship between the working velocity of high speed jet and input pressure is achieved, as demonstrated in Eq. (24).

$$
y_{w v}=0.513 x_{t p}+78.54
$$

where $y_{w v}$ is the working velocity of high speed jet in $\mathrm{m} / \mathrm{s}$.

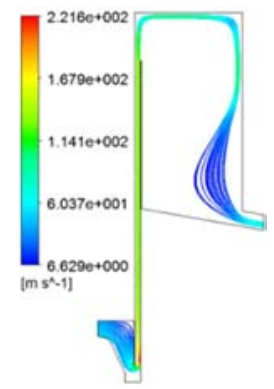

(a) $80 \mathrm{MPa}$

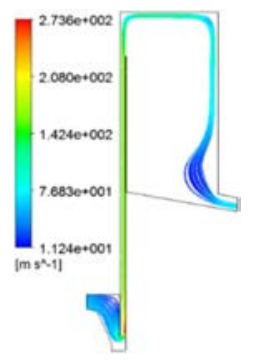

(b) $120 \mathrm{MPa}$

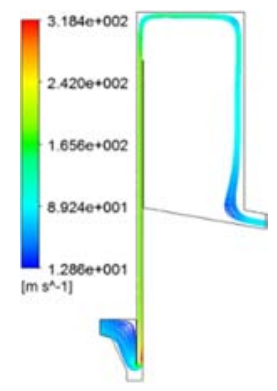

(c) $160 \mathrm{Mpa}$

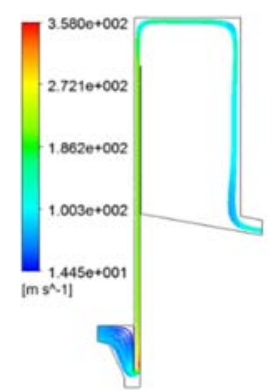

(d) $200 \mathrm{MPa}$

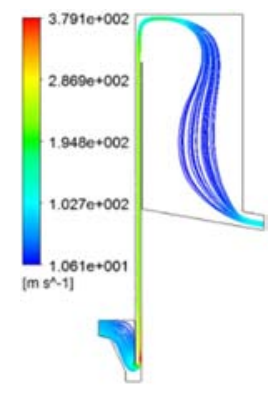

(e) $240 \mathrm{MPa}$

Figure 7. Streamlines of high speed jet under different input pressures.

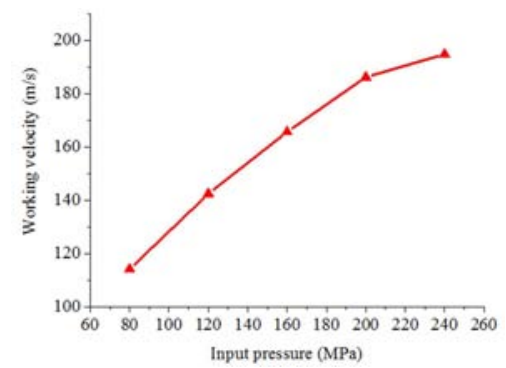

Figure 8. Plot of working velocity versus input pressure.

\section{Conclusions}

Depending on the computational fluid dynamics simulation, the following conclusions are summarized:

(i) When liquid flows through the orifice, the pressure of flows decreases dramatically. By contrast, when liquid flows through the orifice, the velocity of flows soars to the maximum value and then decreases slightly, showing the excellent acceleration performance of the orifice.

(ii) The relationship between the working pressure of high speed jet and input pressure is $y_{w p}=0.1754 x_{i p}-1.136$.

(iii) The relationship between the working velocity of high speed jet and input pressure is $y_{w}=0.513 x_{i p}+78.54$.

(iv) By using Eqs. (23) and (24) obtained in this study, researchers could predict the working pressure and velocity of high speed jet based on their real input pressures.

(v) As the actual operational conditions of high speed jet are overwhelmingly sophisticated, impact factors cannot be fully taken into account in the simulation model. That is, the simulation model is not consummate. In the future, the simulation model will be further revised on the basis of experimental data and the actual operational conditions, in order to reduce the model error. 


\section{Acknowledgements}

The researchers would like to graciously thank Hainan Natural Science Foundation (Project no. 20163113) and the National Natural Science Foundation of China (Project no.31601397).

\section{References}

[1] S. Y. Soon, J. Harbidge, N. J. Titchener-Hooker, and P. A. Shamlou, "Prediction of drop breakage in an ultra-high velocity jet homogenizer," Journal of Chemical Engineering of Japan, vol. 34, no. 5, pp. 640-646, 2001.

[2] Z. Fu, S. J. Luo, J. N. BeMiller, W. Liu, and C. M. Liu, "Influence of high-speed jet on solubility, rheological properties, morphology and crystalline structure of rice starch," Starch/Stärke, vol. 67, pp. 1-9, 2015.

[3] W. Xia, F. Wang, J. H. Li, X. Y. Wei, T. K. Fu, L. H. Cui, T. Li, and Y. F. Liu, "Effect of high speed jet on the physical properties of tapioca starch," Food Hydrocolloids, vol. 49, pp. 35-41, 2015.

[4] Z. Fu, S. J. Luo, J. N. BeMiller, W. Liu, and C. M. Liu, "Effect of high-speed jet on flow behavior, retrogradation, and molecular weight of rice starch," Carbohydrate Polymers, vol. 133, pp. 61-66, 2015.

[5] Z. Fu, S. J. Luo, W. Liu, C. M. Liu, and L. J. Zhan, "Structural changes induced by high speed jet on invitro digestibility and hydroxypropylation of rice starch," International Journal of Food Science \& Technology, vol. 51, pp. 1034-1040, 2016.

[6] H. K. Versteeg and W. Malalasekera, An introduction to computational fluid dynamics: The finite volume method, 2nd Edition, Prentice Hall, Harlow, UK, 2007.

[7] J. H. Ferziger and M. Perić, Computational methods for fluid dynamics, 3rd Edition, Springer, New York, USA, 2002.

[8] Y. F. Fu, J. Gong, P. W. Li, and Z. M. Yang, "Fatigue life assessment of screw blades in screw sand washing machine under extreme load," American Journal of Mechanical Engineering, vol. 5, no. 1, pp. 1-7, 2017.

[9] T. N. Ofei, "Effect of yield power law fluid rheological properties on cuttings transport in eccentric horizontal narrow annulus," Journal of Fluids, vol. 2016, Article ID 4931426, 10 pages, 2016.
[10] Y. H. Yu and Y. Li, "Reynolds-Averaged Navier-Stokes simulation of the heave performance of a two-body floating-point absorber wave energy system," Computers \& Fluids, vol. 73, pp. 104-114, 2013.

[11] H. Xiao, J. L. Wu, J. X. Wang, R. Sun, and C. J. Roy, "Quantifying and reducing model-form uncertainties in Reynolds-averaged Navier-Stokes simulations: A data-driven, physics-informed Bayesian approach," Journal of Computational Physics, vol. 324, pp. 115-136, 2016.

[12] S. J. Kim, J. S. Jung, and S. Kang, "Fully three-dimensional Reynolds-averaged Navier-Stokes modeling for solving free surface flows around coastal drainage gates," Journal of Hydro-environment Research, vol. 13, pp. 121-133, 2016.

[13] V. Yakhot and S. A. Orszag, "Renormalization group analysis of turbulence. I. basic theory," Journal of Scientific Computing, vol. 1, no. 1, pp. 3-51, 1986.

[14] U. Y. Jeong, H. M. Koh, and H. S. Lee, "Finite element formulation for the analysis of turbulent wind flow passing bluff structures using the RNG $k-\varepsilon$ model," Journal of Wind Engineering and Industrial Aerodynamics, vol. 90, pp. 151-169, 2002.

[15] J. J. Kim and J. J. Baik, "A numerical study of the effects of ambient wind direction on flow and dispersion in urban street canyons using the RNG $k-\mathcal{E}$ turbulence model," Atmospheric Environment, vol. 38, pp. 3039-3048, 2004.

[16] V. Yakhot, S. A. Orszag, S. Thangam, T. B. Gatski, and C. G. Speziale, "Development of turbulence models for shear flows by a double expansion technique," Physics of Fluids A Fluid Dynamics, vol. 4, no. 7, pp. 1510-1520, 1992.

[17] D. R. Culver, E. Dowell, D. Smith, Y. Urzhumov, and A. Varghese, "A Volumetric Approach to Wake Reduction: Design, Optimization, and Experimental Verification," Journal of Fluids, vol. 2016, Article ID 3587974, 15 pages, 2016.

[18] W. M. Elnaggar, Z. H. Chen, and Z. G. Huang, "Numerical investigations of body tail projectile," Journal of Applied Science and Engineering, vol. 19, no. 2, pp. 163-168, 2016.

[19] Z. Shang, J. Lou, and H. Y. Li, "Numerical simulation of water jet flow using diffusion flux mixture model," Journal of Fluids, vol. 2014, Article ID 193215, 6 pages, 2014. 